\title{
INCIDENCE OF RECURRENT LARYNGEAL NERVE PALSY IN CERVICAL ANASTOMOSIS AFTER ESOPHAGECTOMY FOR CARCINOMA ESOPHAGUS
}

\author{
Zahid Hussain, Farhan Ahmed Majeed, Maqbool Raza, Aaisha Shahbaz, Atif Rafique, Nabeela Farhan \\ Combined Military Hospital Multan/National University of Medical Sciences (NUMS) Pakistan
}

\begin{abstract}
Objective: To study the incidence of Recurrent Laryngeal Nerve Palsy (RLNP) in cervical anastomosis after esophagectomy for carcinoma of the esophagus.

Place and Duration of Study: Military Hospital's Thoracic surgery departments, Combined Military Hospital Rawalpindi, Combined Military Hospital Lahore and Combined Military Hospital Multan, from Jan 2010 to Sep 2020.

Study Design: Prospective observational study.

Methodology: Designated proformas were used to collect data. Histopathologically proven, operable cases of carcinoma esophagus with normal phonation were included, all of which underwent cervical anastomosis. All cases of benign pathologies and per-operative macroscopically advanced loco-regional disease were excluded. Recurrent laryngeal nerve (RLN) was identified in all cases and follow-up of 6 months for recovery period was executed.

Results: 220 cases were included out of which 121 (55\%) were males while $99(45 \%)$ females. The age range was $14-81$ years (mean is $38.7 \pm 16.78)$. Out of $29(13.6 \%)$ cases underwent minimally invasive esophagectomy (MIE) while thoracophreno laparotomy was performed in 100 (45.4\%) cases, McKeown in 46 (20.9\%) and Trans-hiatal esophagectomy (THE) in 45 (20.4\%) patients. Recurrent Laryngeal Nerve Palsy was found in 19 patients (8.6\%), tracheal injury in $3(1.3 \%)$ and bronchial injury in 1 $(0.4 \%)$ patient. Recurrent Laryngeal Nerve Palsy was transient in 14 cases and permanent damage persisted in 5 patients.

Conclusion: Recurrent Laryngeal Nerve Palsy after esophagectomy is related to increased morbidity due to respiratory complications. With Sharp dissection technique, adequate surgical skill and equipment, the incidence of Recurrent Laryngeal Nerve Palsy can be decreased. In our study, it is less than 8.6\% (transient injury) and 2.2\% (permanent injury).
\end{abstract}

Keywords: Carcinoma esophagus, Esophagectomy, Recurrent laryngeal nerve.

How to Cite This Article: Hussain Z, Majeed FA, Raza M, Shahbaz A, Rafique A, Farhan N. Incidence of Recurrent Laryngeal Nerve Palsy in Cervical Anastomosis After Esophagectomy for Carcinoma Esophagu. Pak Armed Forces Med J 2021; 71 (Suppl-3): S560-564.

Doi: https://doi.org/10.51253/pafmj.v1i1.7937

This is an Open Access article distributed under the terms of the Creative Commons Attribution License (https://creativecommons.org/licenses/by-nc/4.0/), which permits unrestricted use, distribution, and reproduction in any medium, provided the original work is properly cited.

\section{INTRODUCTION}

The incidence of esophageal cancer has been increasing over the last twenty years and currently, it is the 8th most common carcinoma and sixth cause of cancer related death. ${ }^{1-3}$ The treatment modalities being practiced most commonly for resectable tumors is neoadjuvant chemoradiotherapy followed by operative resection with radical lymphadenectomy.4-6 Damage to the Recurrent Laryngeal Nerve (RLN) during esophagectomy is a well-documented complication. The factors contributing to recurrent laryngeal nerve palsy (RLNP) include dissection of cervical paraesophageal and thoracic paratracheal lymph nodes along with thermal injuries, rough handling of the nerve and ischemic injury due to decreased vascular supply to the nerve. ${ }^{7}$ The incidence of RLNP ranges from $0-59 \% .8-15$ This wide disparity is attributed to extent of lymph node dissection, site of anastomosis, surgical technique (two-stage or three-stage), size and stage of tumor,

Correspondence: Dr Farhan Ahmed Majeed, Thoracic Surgery Departments, Combined Military Hospital, Rawalpindi Pakistan method and time of diagnosis of RLN injury. ${ }^{11,16}$

RLN is vital, not only for phonation but for deglutination as well, as it innervates the cricopharyngeus muscle. Therefore patients with RLNP present with hoarseness, aphonia, and dyspnea along with secondary respiratory complications including aspiration pneumonia, atelectasis and acute respiratory distress syndrome (ARDS). Therefore as RLNP is related to increased morbidity, prevention of damage to RLN and its early diagnosis is pivotal during and after esophagectomy respectively. This study has been conducted to evaluate the incidence of RLNP after esophagectomy in patients with resectable esophageal carcinoma and their outcome.

\section{METHODOLOGY}

This prospective observational study was conducted at tertiary care Military Hospitals of Rawalpindi, Lahore and Multan, from Sep 2010 to Sep 2020 by the same surgeon and his supervised team.

Inclusion Criteria: Histopathologically proven cases of carcinoma esophagus were included. 
Exclusion Criteria: All benign pathologies were excluded. Cases that had preoperative hoarseness and per-operative macroscopically advanced loco-regional disease were excluded.

Vocal cords and their mobility was visualized post-operatively with fiberoptic laryngoscopy or bronchoscopy (depending on availability) in patients who had hoarseness. A total of 220 patients of all age groups were included in this study. Ethical approval was sought by the ethical review board. The staging was done according to the American Joint Committee on Cancer (AJCC) classification of esophageal carcinoma with contrast-enhanced computerized tomography of chest and abdomen as the staging tool. Status type of operation performed and follow-up of 6 month period were also recorded for the cases with RLNP. A cervical neck incision was given and dissection was done at the anterior border of sternocleidomastoid muscle, later omohyoid muscle was cut and strap muscles were retracted or cut at the lower end. Middle thyroid vein and inferior thyroid artery were identified and ligated. Diathermy and electrocautery were avoided in bed and the region around the expected track of RLN to avoid thermal injury to the nerve. Sharp dissection was done over the esophagus, parallel to RLN after its identification of nerve in tracheoesophageal groove. Direct traction was avoided by retractors over the nerve.

\section{RESULTS}

A total number of 220 cases were included out of which 121 (55\%) were males while 99 (45\%) were females (Figure-1). The age range was 14-81 years. Out of 29 $(13.6 \%)$ cases underwent minimally invasive esophagectomy (MIE) while thoracophreno laparotomy in 100 cases $(45.4 \%)$, McKeown esophagectomy was done in $46(20.9 \%)$ and Trans-hiatal (THE) in 45 (20.4\%) (TableI). All patients had undergone cervical anastomosis. On histopathology 154 (70\%) were diagnosed to have squamous cell carcinoma while $66(30 \%)$ were adenocarcinoma (Figure-2). RLNP occurred in 19 patients $(8.6 \%)$, tracheal injury in $3(1.3 \%)$ and bronchial injury in $1(0.4 \%)$. Tracheobronchial injury was in the membranous part of the tracheobronchial tree and they did not have RLNP. RLN injury was transient in 14 cases and permanent damage persisted in 5 patients (Figure3). As the nerve was identified, there was $73.6 \%$ clinical recovery over the follow-up of 2-6 months, which was assessed with fiberoptic laryngoscopy. The residual compensated 5 permanent RLNP cases did not require any surgical intervention. Hospital stay of the patients was $>7$ days in all cases of RLNP as compared to normal 4-5 days stay of uneventful procedure. Atelectasis was seen in $9(47 \%)$ patients and responded to rigorous chest physiotherapy. Pneumonia developed in 5 (26\%) cases but none required ventilatory support.

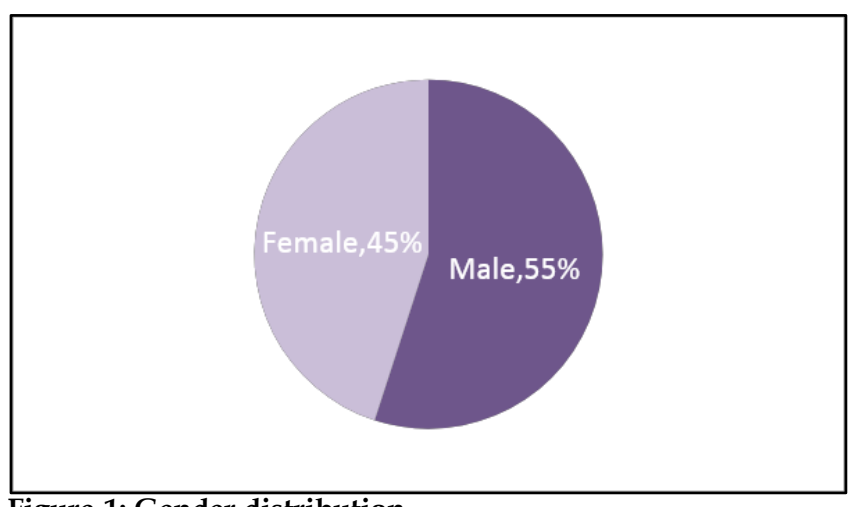

Figure-1: Gender distribution.

Table: Various operative procedures performed for esophagectomy.

\begin{tabular}{l|c|c}
\hline Operative Procedures & Frequency & Percentage \\
\hline McKeown esophagectomy & 46 & 20.9 \\
\hline $\begin{array}{l}\text { Thoraco Phreno Laparotomy, } \\
\text { esophagectomy }\end{array}$ & 100 & 45.4 \\
\hline Trans-hiatal esophagectomy & 45 & 20.4 \\
\hline $\begin{array}{l}\text { Minimal invasive } \\
\text { esophagectomy }\end{array}$ & 29 & 13.6 \\
\hline
\end{tabular}

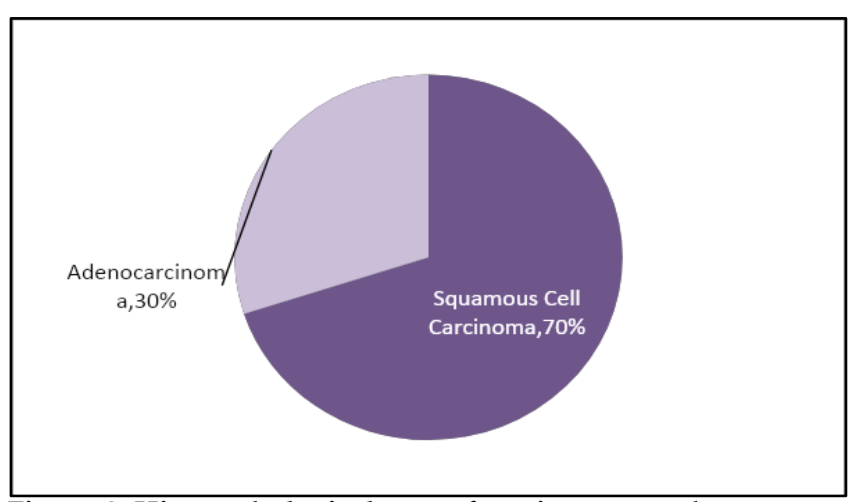

Figure-2: Histopathological type of carcinoma esophagus.

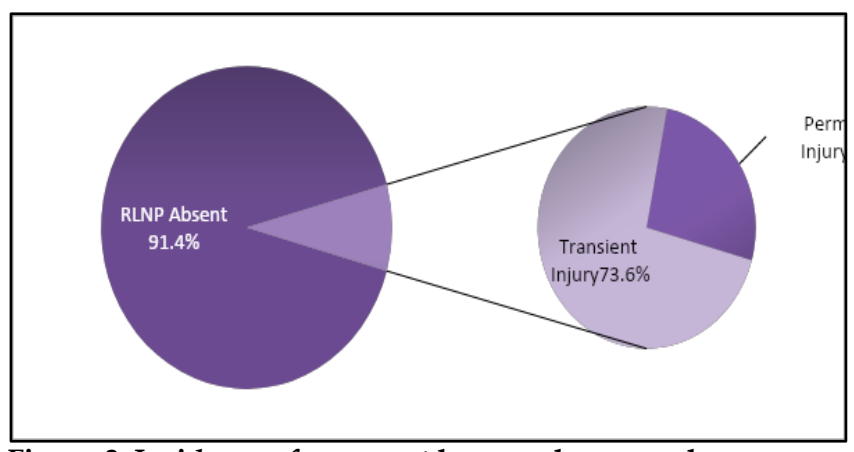

Figure-3: Incidence of recurrent laryngeal nerve palsy. 


\section{DISCUSSION}

RLNP has been defined as any kind of intraoperative damage inflicted to right, left, or both RLN (s) causing paresis or paralysis of the nerve. Paresis has been defined as a partial interruption of laryngeal innervation, leading to weakness of movement, or partial loss of movement, or the impaired movement of laryngeal muscles. Paralysis has been defined as no movement of affected muscles. If the recurrent nerve recovers within 6 months, the RLNP is defined as "transient"; if not, the RLNP is defined as "permanent". ${ }^{17}$

In this study, we found the incidence of RLNP after esophagectomy to be $8.6 \%$ which is in accordance with the results documented in Western studies. In our study unilateral RLNP is limited to left RLNP as during cervical anastomosis left sided neck approach has been used. Scholtemeijeret al reported that out of 451 included patients, $47(10 \%)$ were diagnosed with RLNP. Out of these, almost half recovered fully with more than 6 months of follow-up. ${ }^{18}$ This incidence is in accordance with our study where $72 \%$ recovered in 2 to 6 months duration.

In a study conducted by Gockel et al, from 1985 to 2003, 404 cases of esophagectomy were followed, out of which $60(14.85 \%)$ had RLNP. In 47 out of 60 had unilateral whereas 16 had bilateral RLNP, 12 Pertl et al, found the incidence of URLNP to be 50\% (42/84). Out of 24 cases $(28.6 \%)$ showed a transient URLNP whereas $9(10.7 \%)$ patients had permanent URLNP. The remaining $9(10.7 \%)$ had paresis with loss of follow up. This high incidence of URLNP was attributed to esophagectomy followed by cervical anastomosis, which is not in accordance with our study. ${ }^{14}$

Studies conducted in Japan have found the incidence to be as high as $59 \%$. This is attributed to extensive three-field lymph node dissection. Fujita et al, reported the incidence of RLNP to be $48 \%$ in two-field lymph node dissection in which $32 \%$ had permanent damage ${ }^{19}$. They also reported 70\% RLNP in three-field dissection in which $27 \%$ had permanent damage. In a study conducted in 2015, Koyanagi et al found that out of 782 patients who underwent an esophagectomy with three-field lymph node dissection, simultaneous gastric conduit reconstruction, and cervical anastomosis, RLNP was found in $229(29.3 \%)$ of the patients after esophagectomy: 198 cases had URLNP and 31 bilateral RLNP. ${ }^{13}$ Visbal et al reported an incidence of RLNP to be $0.9 \%$. The reason for such a low incidence was attributed to anastomosis in the chest in the Ivor
Lewis type of esophagectomy. ${ }^{20}$ However in our study, all the anastomoses were in the cervical region.

The incidence being reported is also dependent on the method utilized for RLNP diagnosis. In studies that have utilized clinical assessment and indirect laryngoscopy as diagnostic modalities, the incidence reported is reduced as compared to the studies in which direct visualization of vocal cords through fiberoptic laryngoscopic evaluation is done. ${ }^{13}$ The latter increases the sensitivity of RLNP diagnosis. Earlier and accurate diagnosis of RLNP is essential for decreasing the rate of complications after esophagectomy. In this study, we performed fiberoptic laryngoscopy or bronchoscopy (depending upon the availability) after the clinical diagnosis of hoarseness of voice.

Studies have reported increased incidence of aspiration pneumonia and respiratory complications in patients with RLNP as it is a major motor nerve that innervates the larynx as well as cricopharyngeus muscle, which plays a vital role in swallowing reflex. Therefore RLNP will disrupt the normal mechanisms of respiration, phonation and deglutition. Hence the preservation of RLN will reduce the rate of pulmonary complications which would consequently reduce the chances of reintubation, duration of hospital stay, intensive care unit readmission rates and better quality of life post-surgical intervention. ${ }^{21}$ In our study, hospital admission was prolonged to more than 7 days and $47 \%$ had atelectasis and $26 \%$ incidence of pneumonia was seen in patient with RLNP.

The incidence of RLNP is higher in McKeown esophagectomy as compared to trans-hiatal esophagectomy. This indicates that high mediastinal lymph node dissection results in higher rates of injury. Left-sided RLN is more prone to damage which can be attributed to its longer pathway, close anatomical relation to lymph node station $2 \mathrm{~L}$ and $4 \mathrm{~L}$ as well as damage during esophagogastric anastomosis in which the cervical esophagus is pulled upwards from the left side of the neck. ${ }^{13,16}$ Cases with severe neck infections after the failure of anastomosis and anastomotic leakage are found to have higher rates of RLNP as post-operative edema causes nerve compression. We did not perform neck dissection in our cases therefore a comparison cannot be drawn in this context.

Certain intra-operative modalities have reduced the incidence of RLN injury. These include non invasive intra-operative neurological monitoring (IONM) and reduced use of electro-cautery devices around RLN. IONM is being widely used in thyroid surgery in 
the Western world. Its use enables the surgeon to identify and preserve the RLN. Consequently, it reduces the chances of RLNP and respiratory complications as well. ${ }^{22}$ This facility was not available at our center moreover most cases were intubated with One Lung Ventilation (OLV) tubes during the surgery.

An adequate number of RLNP is caused by thermal injury due to the use of electrocautery devices during esophagectomy. These devices may deliver heat at a single temperature or a varying range of temperatures between 100 and $1200^{\circ} \mathrm{C}$. The reduction of temperature and use of absorbable dense knit hemostat for hemostasis around RLN will significantly reduce the chances of RLNP. ${ }^{23}$ In our operative technique, we avoided the use of diathermy in bed and around the esophagus. Earlier diagnosis of RLNP helps in the reduction of secondary complications. Evaluation of vocal cords at the time of post-surgical endotracheal tube removal, clinical assessment of deglutition process and postponing oral intake reduces the risk of aspiration pneumonia. ${ }^{16}$

Patients with RLNP can benefit from various surgical and non-surgical interventions. Speech and language therapy (SLT) has been shown to increase the quality of life with the achievement of satisfactory function of voice and swallowing. In patients with severe RLNP, methods of management available include intra-cordal injection, type 1 thyroplasty, arytenoid adduction and reconstruction of RLN during esophagectomy should be considered as it restores the thyroarytenoid muscle tone. ${ }^{15}$ The time interval between esophagectomy and operative correction for RLNP has been under consideration. RLNP is usually released in the first post-operative year; therefore earlier surgical intervention is not recommended. As in our study, residual unifocal compensated damage was 2.2\%, $73.6 \%$ percent of the patients improved within the first 6 months of follow-up.

\section{ACKNOWLEDGMENTS}

Special thanks to our supervisor and co-author Prof Farhan Ahmed Majeed for his support and guidance during the process of data collection and discussion. We would also like to extend our gratitude to the administrative departments of Combined Miltary Hospital Rawalpindi, Combined Miltary Hospital Multan and Combined Miltary Hospital Lahore for allowing us to conduct our research in their Thoracic Surgery Department.

\section{LIMITATION OF THE STUDY}

Due to the inavailability of intra-operative neurological monitoring in our surgical departments, this research could not quantify the effect of this modality for the prevention of recurrent laryngeal nerve palsy during esophagectomy.

\section{CONCLUSION}

Recurrent laryngeal nerve palsy is a dreadful complication in esophageal cancer surgery which can play havoc with airway and so with the life. Despite meticulous care and high volume center, the complication may happen and the centers indulged in this kind of surgery need to be adequately equipped for its diagnostic and management capabilities.

\section{Conflict of Interest: None.}

\section{Authors' Contribution}

$\mathrm{ZH}$ : conceived and designed the research, wrote the article and collected the data. FAM: conceived and designed the research, supervised the data collection, data analysis, aided in interpretting the results and approved the version to be published, MR: contributed in data and analysis tools, AS: wrote the discussion with contribution from all the authors, did the referencing and final editing of the article, AR: provided critical feedback and helped in shaping the article, NF: revised the article content.

\section{REFERENCES}

1. Bray F, Ferlay J, Soerjomataram I, Siegel RL, Torre LA, Jemal A. Global cancer statistics 2018: Globocan estimates of incidence and mortality worldwide for 36 cancers in 185 countries. CA Cancer J Clin 2018; 68(6): 394-424.

2. Then EO, Michell Lopez SS, Gayam V, Sunkara T, Culliford A, Gaduputi V. Esophageal cancer: an updated surveillance epidemiology and end results database analysis. World J Oncol 2020; 11(2): 55-58.

3. Uhlenhopp DJ, Then EO, Sunkara T, Gaduputi V. Epidemiology of esophageal cancer: update in global trends, etiology and risk factors. Clin J Gastroenterol 2020: 2(1): 1-12.

4. Schizas D, Lazaridis II, Moris D, Mastoraki A, Lazaridis L-D, Tsilimigras DI, et al. The role of surgical treatment in isolated organ recurrence of esophageal cancer-a systematic review of the literature. World J Surg Oncol 2018; 16(1): 1-10.

5. Tu C-C, Hsu PK. The frontline of esophageal cancer treatment: questions to be asked and answered. Ann Transl Med 2018; 6(4): $1-5$.

6. Watanabe M, Otake R, Kozuki R, Toihata T, Takahashi K, Okamura A, et al. Recent progress in multidisciplinary treatment for patients with esophageal cancer. Surg Today 2020; 50(1): 1220.

7. Myssiorek D. Recurrent laryngeal nerve paralysis: anatomy and etiology. Otolaryngol Clin North Am 2004; 37(1): 25-44.

8. Gong L, Jiang H, Yue J, Duan X, Tang P, Ren P, et al. Comparison of the short-term outcomes of robot-assisted minimally invasive, video-assisted minimally invasive, and open esophagectomy. J Thorac Dis 2020; 12(3): 916-920.

9. Shah R, Hammoud Z. Comments on: lower incidence of postoperative pulmonary complications following robot-assisted minimally invasive esophagectomy for esophageal cancer: propensity score-matched comparison to conventional minimally invasive esophagectomy. Ann Surg Oncol 2021; 28(2): 594-595.

10. van Workum F, Berkelmans GH, Klarenbeek BR, Nieuwenhuijzen GA, Luyer MD, Rosman C. McKeown or Ivor Lewis totally minimally invasive esophagectomy for cancer of the esophagus and gastroesophageal junction: systematic review and metaanalysis. J Thorac Dis 2017; 9(Suppl-8): S826. 


\section{Recurrent Laryngeal Nerve Palsy}

11. Gelpke H, Grieder F, Decurtins M, Cadosch D. Recurrent laryngeal nerve monitoring during esophagectomy and mediastinal lymph node dissection. World J Surg 2010; 34(10): 23792382.

12. Gockel I, Kneist W, Keilmann A, Junginger T. Recurrent laryngeal nerve paralysis (RLNP) following esophagectomy for carcinoma. Eur J Surg Oncol 2005; 31(3): 277-281.

13. Koyanagi K, Igaki H, Iwabu J, Ochiai H, Tachimori Y. Recurrent laryngeal nerve paralysis after esophagectomy: respiratory complications and role of nerve reconstruction. Tohoku J Exp Med 2015; 237(1): 1-8.

14. Pertl L, Zacherl J, Mancusi G, Gächter J, Asari R, Schoppmann S, et al. High risk of unilateral recurrent laryngeal nerve paralysis after esophagectomy using cervical anastomosis. Eur Arch Otorhinolaryngol 2011; 268(11): 1605-1610.

15. Wright CD, Zeitels SM. Recurrent laryngeal nerve injuries after esophagectomy. Thorac Surg Clin 2006; 16(1): 23-33.

16. Taniyama Y, Miyata G, Kamei T, Nakano T, Abe S, Katsura K, et al. Complications following recurrent laryngeal nerve lymph node dissection in oesophageal cancer surgery. Interact Cardiovasc Thorac Surg 2015; 20(1): 41-46.

17. Rubin AD, Sataloff RT. Vocal fold paresis and paralysis. Otolaryngol Clin North Am 2007; 40(5): 1109-1131.
18. Scholtemeijer MG, Seesing MF, Brenkman HJ. Recurrent laryngeal nerve injury after esophagectomy for esophageal cancer: incidence, manage-ment, and impact on short-and long-term outcomes. J Thorac Dis 2017; 9(Suppl-8): S868.

19. Fujita H, Kakegawa T, Yamana H, Shima I, Toh Y. Mortality and morbidity rates, postoperative course, quality of life, and prognosis after extended radical lymphadenectomy for esophageal cancer. Comparison of three-field lymphadenectomy with twofield lymphadenectomy. Ann Surg 1995; 222(5): 654-658.

20. Visbal AL, Allen MS, Miller DL, Deschamps C, Trastek VF, Pairolero PC. Ivor Lewis esophagogastrectomy for esophageal cancer. Ann Thorac Surg 2001; 71(6): 1803-1808.

21. Oshikiri T, Takiguchi G, Hasegawa H, Yamamoto M, Kanaji S, Yamashita $\mathrm{K}$, et al. Postoperative recurrent laryngeal nerve palsy is associated with pneumonia in minimally invasive esophagectomy for esophageal cancer. Surg Endosc 2021; 35(2): 1-5.

22. Yuda M, Nishikawa K, Ishikawa Y, Takahashi K. Intraoperative nerve monitoring during esopha-gectomy reduces the risk of recurrent laryngeal nerve palsy. Surg Endosc 2021: 5(2): 1-8.

23. Rino $Y$, Yukawa N, Sato T, Yamamoto N, Tamagawa H. Using NU-KNIT for hemostasis around recurrent laryngeal nerve during transthoracic esophagectomy with lymphadenectomy for esophageal cancer. BMC Res Notes 2014; 7(1): 1-4. 